\title{
High-grade dysplasia associated with fundic gland polyposis in a familial adenomatous polyposis patient, with special reference to $A P C$ mutation profiles
}

Shigeki Sekine ${ }^{1}$, Tadakazu Shimoda $^{2}$, Satoshi Nimura ${ }^{2}$, Yukihiro Nakanishi ${ }^{1}$, Takayuki Akasu $^{3}$, Hitoshi Katai ${ }^{3}$, Takuji Gotoda ${ }^{4}$, Tatsuhiro Shibata ${ }^{1}$, Michiie Sakamoto ${ }^{1,5}$ and Setsuo Hirohashi ${ }^{1}$

${ }^{1}$ Pathology Division, National Cancer Center Research Institute, Tokyo, Japan; ${ }^{2}$ Clinical Laboratory Division; ${ }^{3}$ Department of Surgery; ${ }^{4}$ Department of Endoscopy, National Cancer Center Hospital, Tokyo, Japan and

${ }^{5}$ Department of Pathology, Keio University School of Medicine, Tokyo, Japan

\begin{abstract}
We report a patient with familial adenomatous polyposis who developed high-grade dysplasia against a background of fundic gland polyposis. Two large high-grade dysplasia lesions were found in the gastric body, where numerous fundic gland polyps were present. In both lesions, the dysplastic epithelium covered nonneoplastic oxyntic glands that occasionally exhibit cystic changes. A genetic analysis for APC (adenomatous polyposis coli) revealed a somatic 50-bp deletion involving codons 1502-1517 and 2-bp deletion at codon 1465 in each lesion of high-grade dysplasia. In contrast, six of the 18 fundic gland polyps were found to harbor an identical mutation: 1-bp insertion at codon 1556. Both lesions of high-grade dysplasia and the fundic gland polyps were similarly located in the fundic gland area and were caused by the inactivation of $A P C$; however, their mutation profiles of $A P C$ were different. These results imply that fundic gland polyps and high-grade dysplasia of the stomach have distinct preferences for APC genotypes in their development. Modern Pathology (2004) 17, 1421-1426. doi:10.1038/modpathol.3800178
\end{abstract}

Keywords: gastric dysplasia; familial adenomatous polyposis; APC

Fundic gland polyp is the most common type of gastric polyp and has long been thought to be a hamartomatous lesion with no malignant potential. Fundic gland polyp occurs sporadically or in association with familial adenomatous polyposis (FAP). Fundic gland polyps are found in $26-43 \%$ of FAP patients, and tend to be more numerous in FAP-associated cases. ${ }^{1-3}$ FAP patients carry germline mutations of $A P C$ (adenomatous polyposis coli), and tumors associated with FAP develop through the biallelic inactivation of $A P C$ by germline and somatic mutations. Previous studies have revealed the presence of somatic $A P C$ mutations in FAPassociated fundic gland polyps, indicating that FAPassociated fundic gland polyps are a neoplastic lesion caused by the inactivation of both $A P C$

Correspondence: S Hirohashi, MD, Pathology Division, National Cancer Center Research Institute, 5-1-1 Tsukiji, Chuo-ku, Tokyo 104-0045, Japan.

E-mail: shirohas@ncc.go.jp

Received 7 February 2004; revised and accepted 21 April 2004 alleles, similar to the mechanism of other FAPassociated tumors. ${ }^{4,5}$

FAP-associated fundic gland polyps have been reported to frequently develop dysplasia in their foveolar epithelium, whereas this rarely occurs in sporadic fundic gland polyps. ${ }^{5,6}$ However, even in FAP-associated fundic gland polyps, the occurrence of adenocarcinoma or high-grade dysplasia is extremely rare. We present an FAP patient who developed two large high-grade dysplasia lesions against a background of fundic gland polyposis and report the results of a genetic analysis for $A P C$ mutations.

\section{Case report}

A male patient was diagnosed as suffering from FAP and underwent a colectomy in another hospital when he was 28 years old. His niece had also been diagnosed as having FAP, and his mother and brother had died of colon cancer at the ages of 39 and 41 years, respectively. The patient had been 
followed up at our hospital using upper and lower gastrointestinal endoscopy examinations since he was 37 years old. Fundic gland polyposis was detected endoscopically, but no adenomas/dysplasia or carcinomas were found in his stomach or duodenum. When the patient was 46 years old, a biopsy specimen obtained from his gastric body showed high-grade dysplasia (corresponding to Category 4 in the Vienna classification ${ }^{7}$ ), and a proximal gastrectomy was performed. A germline $A P C$ mutation has not been tested so far.

\section{Materials and methods}

The resected sample were routinely fixed with $10 \%$ formalin and embedded in paraffin. The genetic study was approved by the ethics committee for human genome analysis research of the National Cancer Center, Japan, and was performed after receiving the subject's written informed consent. Routinely processed and paraffin-embedded archival materials were used for the analysis. The sections were stained briefly with hematoxylin and eosin, and the dysplastic epithelium and foveolar epithelium of the fundic gland polyps were dissected using sterilized toothpicks under a microscope. DNA extraction was performed as described previously. ${ }^{8}$ For lesions 1 and 2, three samples were obtained from separate areas of each lesion.

For the $A P C$ analysis, the samples were subjected to polymerase chain reaction (PCR) with five pairs of primers encompassing the mutation cluster region of $A P C$ (S-1-s: 5' - GAAATAGGATGTAATCAG ACG-3' and S-1-as: 5'- CGCTCCTGAAGAAAATTC AAC-3'; S-2-s: 5' - ACTGCAGGGTTCTAGTTTATC-3' and S-2-as: $5^{\prime}-$ GAGCTGGCAATCGAACGACT-3'; S-3-s: $5^{\prime}-$ TACTTCTGTCAGTTCACTTGATA-3' and S-3-as: 5'- ATTTTTAGGTACTTCTCGCTTG-3'; S-4-s: $5^{\prime}$-AAACACCTCCACCACCTCC-3' ${ }^{\prime}$ and S-4-as: 5'-GCATTATTCTTAATTCCACATC-3'; S-5-s: 5'-TGA

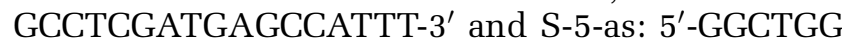
CTTTTTTGCTTTAC- $3^{\prime}$ ). Analysis of $\beta$-catenin was performed by using a previously described pair of primers encompassing exon 3 of $\beta$-catenin. ${ }^{8}$ PCR and direct sequencing were performed as described previously. ${ }^{8}$ Each experiment, including the DNA extraction, was performed at least twice. In samples with mutations, the PCR products were subcloned into pCR2.1 plasmid vectors using a TOPO TA cloning kit (Invitrogen, Carlsbad, CA, USA). Several clones were sequenced in each lesion to isolate the mutant and wild-type alleles.

\section{Results}

Grossly, two large, almost flat lesions with relatively well-demarcated areas of mucosal irregularity and measuring $70 \times 48 \mathrm{~mm}$ and $44 \times 38 \mathrm{~mm}$, respectively, were identified (lesions 1 and 2; Figure 1).

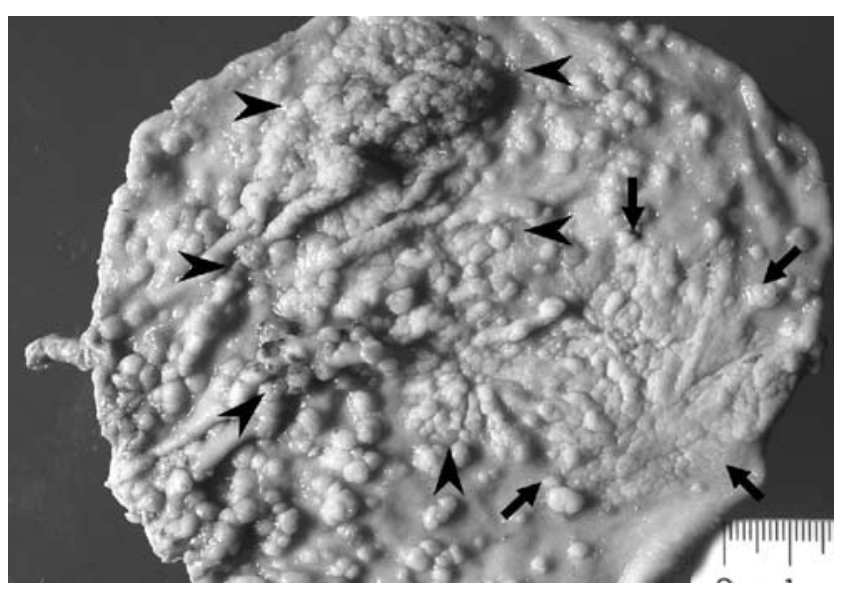

Figure 1 Resected specimen obtained by proximal gastrectomy. Two lesions appear as mucosal irregularities on a background of diffuse polyposis (lesion 1, arrowheads; lesion 2, arrows). Lesion 1 partly exhibits papillary surface (upper side).

Lesion 1 had a focal elevated area with papillary surface. Numerous sessile polyps of up to $6 \mathrm{~mm}$ were seen throughout the specimen.

Microscopically, lesions 1 and 2 showed highgrade dysplasia. We did not diagnose these lesions as adenomas because they were mostly flat. In both lesions, the dysplastic epithelium exhibited papillary growth and covered non-neoplastic oxyntic glands with occasional cystic changes (Figure 2a, b). The tumor cells had large oval atypical nuclei showing pseudostratification (Figure 3a, b). Two small-sized low-grade dysplasia lesions, measured 8 and $6 \mathrm{~mm}$, were found lying on thin oxyntic glands (Figure 2c). These lesions showed a proliferation of atypical glands resembling foveolar epithelium and had basally located nuclei with mild atypia (Figure 3c). The other small polyps were typical fundic gland polyps with cystically dilated oxyntic glands (Figure 2d).

By genetic analysis, lesion 1 was found to harbor a 50-bp deletion involving codons 1502-1517, resulting in the formation of a stop codon at the junction (Figure 4). Lesion 2 had a 2-bp deletion involving codon 1465. In each lesion, samples obtained from three separate areas showed the same mutation. Six out of 18 fundic gland polyps harbored an identical mutation: 1-bp insertion at codon 1556. No genetic alterations were found in the two lesions of lowgrade dysplasia or in 12 of the 18 fundic gland polyps. There were no histological differences between fundic gland polyps with and without mutations. A germline mutation was not identified within the mutation cluster region. No $\beta$-catenin mutations were identified in any of the lesions.

\section{Discussion}

FAP is characterized by the occurrence of multiple adenomas with malignant potential in the colorectum. However, extracolonic manifestations are 

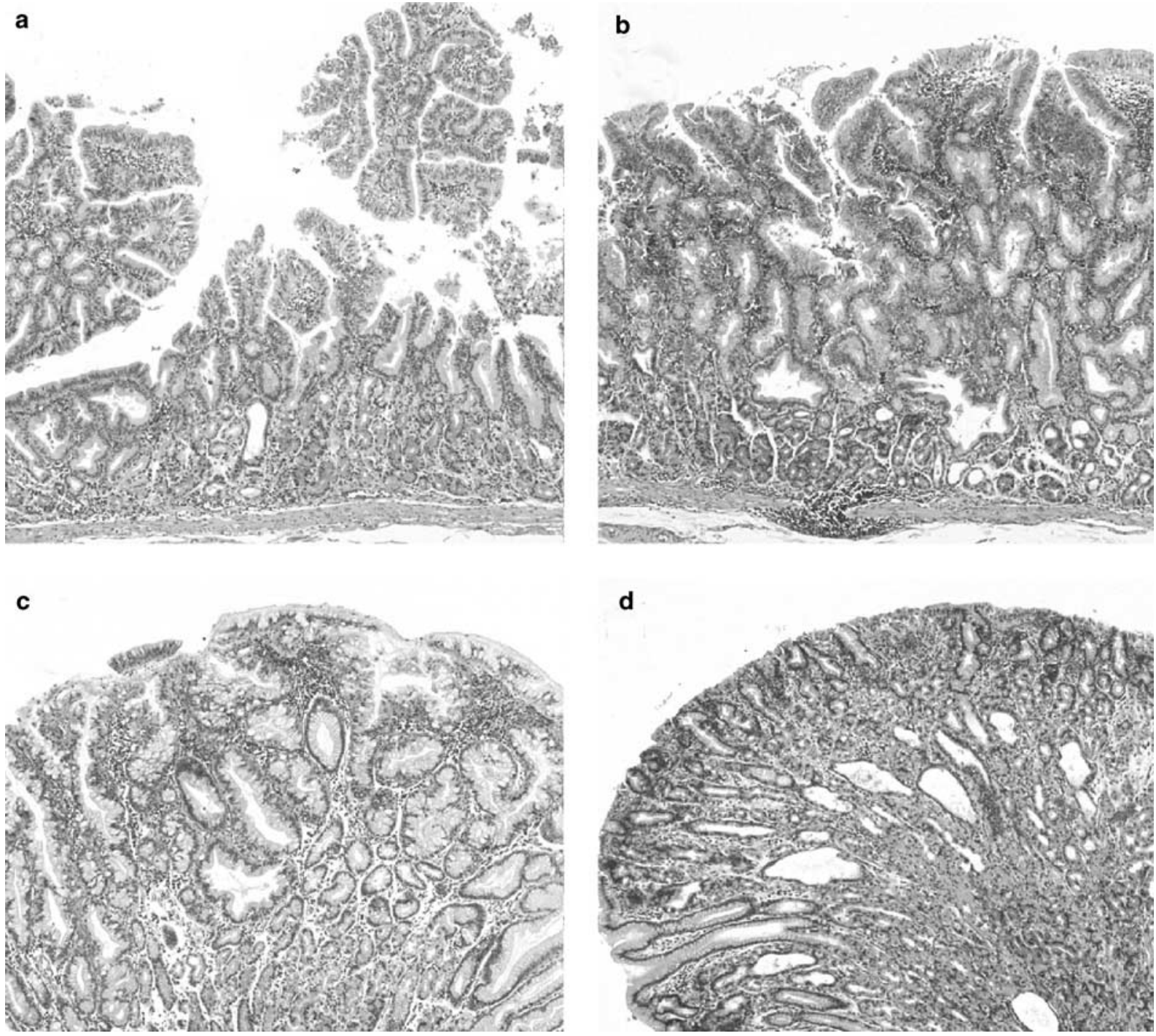

Figure 2 Histological appearances of the lesions. Histology of the high-grade dysplasia (lesion 1, a; lesion 2, b) and low-grade dysplasia (c) lesions. A dysplastic epithelium covers the oxyntic glands with occasional cystic changes (a, b). Fundic gland polyp showing cystic changes in its glands (d).

also known to occur. Fundic gland polyp is the most common gastric complication of FAP and has long been thought to have no malignant potential. ${ }^{1-3}$ Although some reports have described the frequent association of low-grade dysplasia in FAPassociated fundic gland polyps, ${ }^{5,6}$ there have been only several single case reports describing highgrade dysplasia or adenocarcinoma in association with fundic gland polyposis. ${ }^{9-13}$ These reports suggest that FAP-associated fundic gland polyp is a potential premalignant lesion, although malignant progression is quite rare.

In the present case, two large high-grade dysplasia lesions were found in the upper portion of the gastric body where numerous fundic gland polyps were present. The dysplastic epithelium covered bland-looking oxyntic glands with occasional cystic changes in both lesions. These histological findings resembled those of fundic gland polyps with dysplasia, although the size of lesions was quite different. Based on their location and histological findings, we believe that these dysplastic lesions may have arisen from fundic gland polyps. Large, flat dysplastic lesions, as seen in the present case have not been previously described in association with fundic gland polyposis. Histologically, these lesions would fall between fundic gland polyps with focal foveolar dysplasia and invasive cancer in fundic gland polyp-related carcinogenesis.

The genetic analysis revealed somatic $A P C$ mutations in both high-grade dysplasia lesions and in six of 18 fundic gland polyps. Although a germline mutation has not been identified in this patient, most clinically diagnosed FAP patients are thought to have germline $A P C$ mutations. ${ }^{14}$ The high frequency of somatic $A P C$ mutations and the absence of $\beta$-catenin mutation in fundic gland polyps is also suggestive of the presence of a germline $A P C$ 

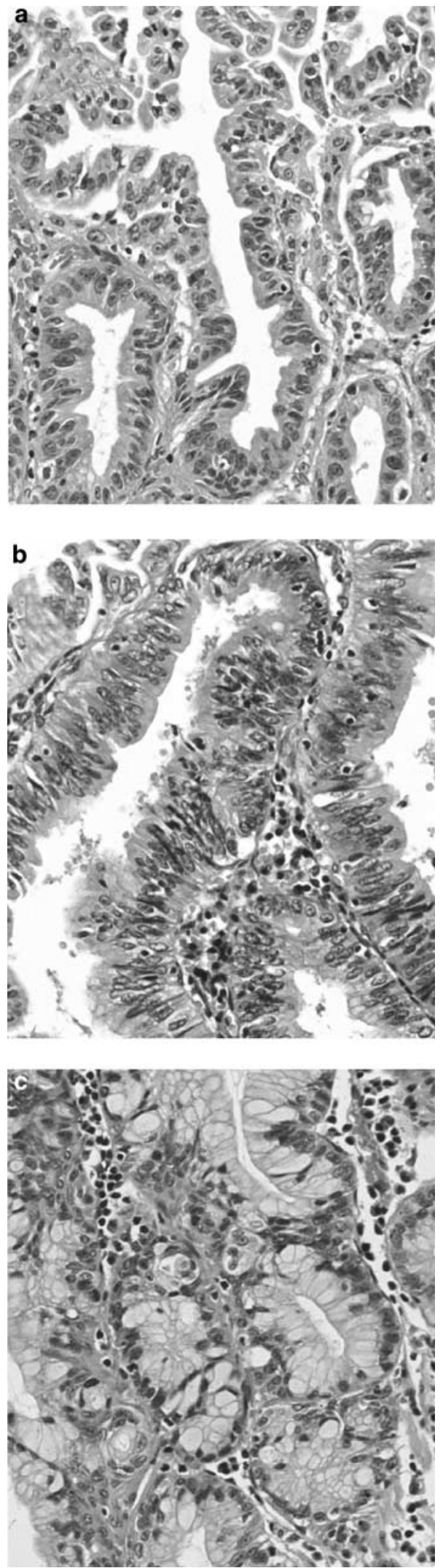

Figure 3 High-power view of the dysplastic lesions. Lesions 1 (a) and 2 (b) exhibit high-grade dysplasia. Note the large pseudostratified nuclei with prominent nucleoli. Low-grade dysplasia (c). Basally located nuclei with mild atypia.

mutation, since most sporadic fundic gland polyps harbor a $\beta$-catenin mutation, with $A P C$ mutations being quite rare. ${ }^{5,8,15}$ Thus, the present patient probably had a germline $A P C$ mutation located outside of the mutation cluster region. The presence of somatic APC mutations in the lesions of highgrade dysplasia indicates that they developed as an FAP-related complication through the biallelic inactivation of $A P C$.

Interestingly, the high-grade dysplasia lesions and the fundic gland polyps had different $A P C$ mutation profiles. All mutations found in the fundic gland polyps were identical: 1-bp insertion at codon 1556. This result agrees with previous reports showing that mutations involving codon 1556 constituted most of the $A P C$ mutations in fundic gland polyps. ${ }^{4,5,16}$ On the other hand, both of the mutations found in the high-grade dysplasia lesions were located in a more $5^{\prime}$-region. The tumorsuppressive effect of $A P C$ is thought to depend mainly on its activity in $\beta$-catenin downregulation, ${ }^{17}$ and it has been recently suggested that truncated $A P C$ may partially retain its function. ${ }^{18-20}$ The $A P C$ protein contains seven 20-amino-acid repeat motifs that are involved in $\beta$-catenin downregulation. The number of retained 20-amino-acid repeats in truncated APC is correlated with its retained ability to influence $\beta$-catenin downregulation, and $A P C$ mutations might be selected to obtain an optimal level of $\beta$-catenin accumulation in the process of tumorigenesis. ${ }^{21,22}$ The optimal level of $\beta$-catenin seems to depend on the tumor type, because the mutational hot spots in $A P C$ are known to differ according to the type of tumor. For example, colorectal tumors preferentially harbor mutations resulting in a truncated protein with one or two 20 -amino-acid repeats. ${ }^{21-23}$ On the other hand, desmoid tumors tend to have a truncated protein with two or three 20 -amino-acid repeats. ${ }^{23}$

Regarding the present case, the selection mechanisms for the $A P C$ mutations in the fundic gland polyp and high-grade dysplasia lesions were probably different. The 1-bp insertion at codon 1556, which was found in six of 18 fundic gland polyps, results in the formation of truncated APC with three 20 -amino-acid repeats. In contrast, both mutations found in the high-grade dysplasia lesions result in the formation of a truncated APC with two intact 20amino-acid repeats. One possible explanation for this result is that the high-grade dysplasia lesions and the fundic gland polyps are histogenetically unrelated because their APC mutation profiles were different. However, their topographically close association and the histological findings seem to indicate a histogenetic linkage. The selection of a truncated APC protein with three 20-amino-acid repeats seems to be the preferred but not strict requirement since a small number of fundic gland polyps have been reported to harbor truncated APC proteins with two 20 -amino-acid repeats. ${ }^{4,5}$ 

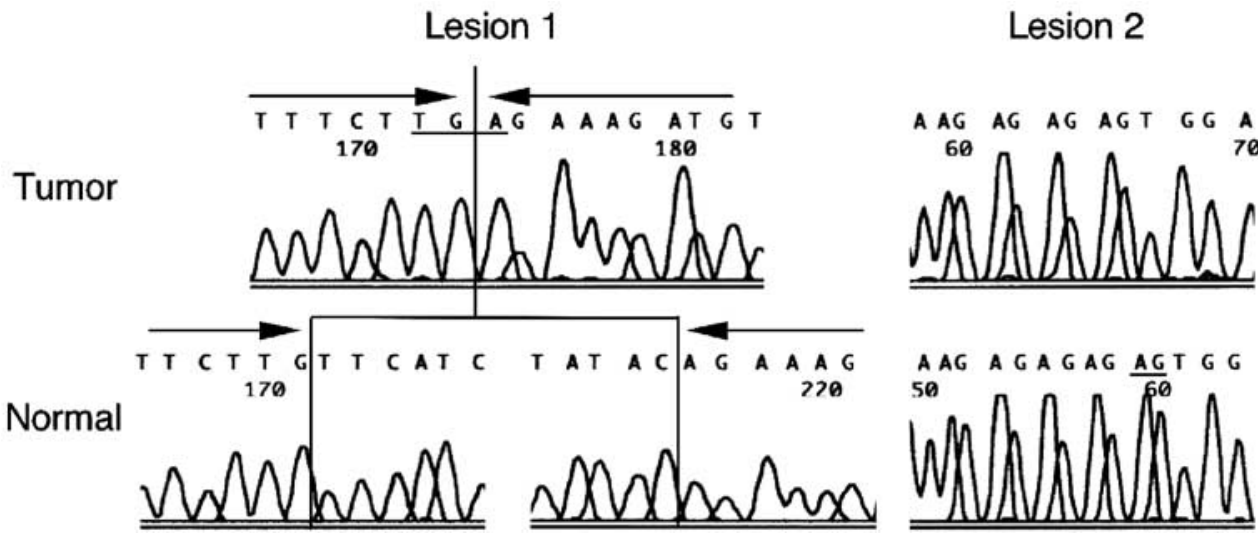

Figure 4 Mutational $A P C$ analysis. Lesion 1 has a 50-bp deletion involving codons 1502-1517, resulting in the formation of a stop codon at the junction (underlined). Lesion 2 has 2-bp deletion at codon 1465. A single nucleotide insertion at codon 1556 in one of the fundic gland polyps.

These facts may be explained by an alternative hypothesis that we would like to propose here: the acquisition of a truncated APC with three 20-aminoacid repeats leads to the formation of fundic gland polyps, which do not progress to high-grade dysplasia; the acquisition of truncated APC with two 20-amino-acid-repeats rarely leads to the formation of fundic gland polyps, but when it does, these fundic gland polyps are more likely to progress to high-grade dysplasia.

The retention of a large number of 20-amino-acid repeats has been suggested to be optimal for tumorigenesis in upper gastrointestinal tumors compared with colonic tumors, in FAP patients. ${ }^{24}$ Nevertheless, the present study implies that the selection mechanisms for $A P C$ mutations are more complex than previously thought, and that different types of tumors have distinct preferences for specific $A P C$ mutations in gastric tumorigenesis.

\section{Acknowledgements}

This work was supported by Grant-in-Aid for Second Term Comprehensive 10-year Strategy for Cancer Control from the Ministry of Health, Labour and Welfare, Japan.

\section{References}

1 Iida $\mathrm{M}$, Yao $\mathrm{T}$, Itoh $\mathrm{H}$, et al. Natural history of fundic gland polyposis in patients with familial adenomatosis coli/Gardner's syndrome. Gastroenterology 1985;89: 1021-1025.

2 Sarre RG, Frost AG, Jagelman DG, et al. Gastric and duodenal polyps in familial adenomatous polyposis: a prospective study of the nature and prevalence of upper gastrointestinal polyps. Gut 1987;28:306-314.

3 Domizio P, Talbot IC, Spigelman AD, et al. Upper gastrointestinal pathology in familial adenomatous polyposis: results from a prospective study of 102 patients. J Clin Pathol 1990;43:738-743.

4 Toyooka M, Konishi M, Kikuchi-Yanoshita R, et al. Somatic mutations of the adenomatous polyposis coli gene in gastroduodenal tumors from patients with familial adenomatous polyposis. Cancer Res 1995;55: 3165-3170.

5 Abraham SC, Nobukawa B, Giardiello FM, et al. Fundic gland polyps in familial adenomatous polyposis: neoplasms with frequent somatic adenomatous polyposis coli gene alterations. Am J Pathol 2000; 157:747-754.

$6 \mathrm{Wu}$ TT, Kornacki S, Rashid A, et al. Dysplasia and dysregulation of proliferation in foveolar and surface epithelia of fundic gland polyps from patients with familial adenomatous polyposis. Am J Surg Pathol 1998;22:293-298.

7 Schlemper RJ, Riddell RH, Kato Y, et al. The Vienna classification of gastrointestinal epithelial neoplasia. Gut 2000;47:251-255.

8 Sekine S, Shibata T, Yamauchi Y, et al. $\beta$-Catenin mutations in sporadic fundic gland polyps. Virchows Arch 2002;440:381-386.

9 Coffey Jr RJ, Knight Jr CD, van Heerden JA, et al. Gastric adenocarcinoma complicating Gardner's syndrome in a North American woman. Gastroenterology 1985;88:1263-1266.

10 Odze RD, Quinn PS, Terrault NA, et al. Advanced gastroduodenal polyposis with ras mutations in a patient with familial adenomatous polyposis. Hum Pathol 1993;24:442-448.

11 Zwick A, Munir M, Ryan CK, et al. Gastric adenocarcinoma and dysplasia in fundic gland polyps of a patient with attenuated adenomatous polyposis coli. Gastroenterology 1997;113:659-663.

12 Hofgartner WT, Thorp M, Ramus MW, et al. Gastric adenocarcinoma associated with fundic gland polyps in a patient with attenuated familial adenomatous polyposis. Am J Gastroenterol 1999;94: 2275-2281.

13 Attard TM, Giardiello FM, Argani P, et al. Fundic gland polyposis with high-grade dysplasia in a child with attenuated familial adenomatous polyposis and familial gastric cancer. J Pediatr Gastroenterol Nutr 2001;32:215-218. 
14 Laken SJ, Papadopoulos N, Petersen GM, et al. Analysis of masked mutations in familial adenomatous polyposis. Proc Natl Acad Sci USA 1999;96: 2322-2326.

15 Abraham SC, Nobukawa B, Giardiello FM, et al. Sporadic fundic gland polyps: common gastric polyps arising through activating mutations in the $\beta$-catenin gene. Am J Pathol 2001;158:1005-1010.

16 Abraham SC, Park SJ, Mugartegui L, et al. Sporadic fundic gland polyps with epithelial dysplasia: evidence for preferential targeting for mutations in the adenomatous polyposis coli gene. Am J Pathol 2002;161:1735-1742.

17 Sparks AB, Morin PJ, Vogelstein B, et al. Mutational analysis of the APC/ $\beta$-catenin/Tcf pathway in colorectal cancer. Cancer Res 1998;15; 58:1130-1134.

18 Henderson BR. Nuclear-cytoplasmic shuttling of APC regulates $\beta$-catenin subcellular localization and turnover. Nat Cell Biol 2000;2:653-660.

19 Kielman MF, Rindapaa M, Gaspar C, et al. APC modulates embryonic stem-cell differentiation by controlling the dosage of $\beta$-catenin signaling. Nat Genet 2002;32:594-605.
20 Rosin-Arbesfeld R, Cliffe A, Brabletz T, et al. Nuclear export of the APC tumour suppressor controls $\beta$-catenin function in transcription. EMBO J 2003;22:1101-1113.

21 Albuquerque C, Breukel C, van der Luijt R, et al. The 'just-right' signaling model: APC somatic mutations are selected based on a specific level of activation of the $\beta$-catenin signaling cascade. Hum Mol Genet 2002;11: 1549-1560.

22 Rowan AJ, Lamlum H, Ilyas M, et al. APC mutations in sporadic colorectal tumors: a mutational "hotspot" and interdependence of the "two hits". Proc Natl Acad Sci USA 2000;97:3352-3357.

23 Lamlum $\mathrm{H}$, Ilyas $\mathrm{M}$, Rowan $\mathrm{A}$, et al. The type of somatic mutation at APC in familial adenomatous polyposis is determined by the site of the germline mutation: a new facet to Knudson's 'two-hit' hypothesis. Nat Med 1999;5:1071-1075.

24 Groves C, Lamlum H, Crabtree M, et al. Mutation cluster region, association between germline and somatic mutations and genotype-phenotype correlation in upper gastrointestinal familial adenomatous polyposis. Am J Pathol 2002;160: 2055-2061. 